\title{
Degradation Kinetics of Glucuronic Acid in Subcritical Water
}

\author{
Rongchun Wang, ${ }^{1}$ Tze Loon Neoh, ${ }^{1}$ Takashi Kobayashi, ${ }^{1}$ Yasuhito Miyake, ${ }^{2}$ Asao Hosoda, ${ }^{2}$ \\ Hisaji TANiguchi, ${ }^{3}$ and Shuji Adachi, ${ }^{1, \dagger}$ \\ ${ }^{1}$ Division of Food Science and Biotechnology, Graduate School of Agriculture, Kyoto University, \\ Sakyo-ku, Kyoto 606-8502, Japan \\ ${ }^{2}$ Industrial Technology Center of Wakayama Prefecture, 60 Ogura, Wakayama 649-6261, Japan \\ ${ }^{3}$ Wakayama Industry Promotion Foundation, 60 Ogura, Wakayama 649-6261, Japan
}

Received November 6, 2009; Accepted December 26, 2009; Online Publication, March 7, 2010

[doi:10.1271/bbb.90818]

The degradation kinetics of glucuronic acid (GlcA) under subcritical conditions from 160 to $200^{\circ} \mathrm{C}$ was studied in a continuous tubular reactor. The formation of glucuronolactone (GICL) during the treatment of GlcA in subcritical water was substantiated by ESITOF-MS and ${ }^{1} \mathrm{H}$ NMR. The degradation of GlcA consisted of the reversible conversion of GlcA to GIcL and the irreversible degradation of the two compounds. The changes in the concentrations of GlcA and GIcL with residence time could be described by first-order kinetics. Higher temperatures accelerated the degradation of GlcA, and thus resulted in rises in the $\mathrm{pH}$ value. The degradation reaction of GlcL under the same conditions was also investigated. The activation energy of the reverse hydrolysis of GIcA to GIcL and that of the hydrolysis of GlcL to GlcA were determined to be 88.5 and $63.2 \mathrm{~kJ} / \mathrm{mol}$ respectively. The enthalpy change in the reversible conversion between GlcA and GlcL was $25.4 \mathrm{~kJ} / \mathrm{mol}$.

Key words: glucuronic acid; glucuronolactone; degradation; subcritical water; kinetics

Subcritical water refers to water at elevated temperatures below $374{ }^{\circ} \mathrm{C}$ that remains in a liquid state due to sufficient compression. The use of subcritical water as a reaction medium has several advantages, including improved safety and environmental friendliness. Clifford $^{1)}$ related the usefulness of subcritical water to its two characteristic features of lower dielectric constant and higher ion product relative to ambient water. The lower dielectric constant of subcritical water enables it to dissolve hydrophobic substances, ${ }^{2-4)}$ while the increase in ion product, by up to 3 orders of magnitude, owing to temperature increase renders subcritical water capable of acting as an acid or base catalyst for hydrolysis and degradation. ${ }^{5-7)}$ Thus the use of subcritical water can also be regarded as a potential replacement for environmentally undesirable acid catalysts. On account of these advantages, subcritical water has increasingly received attention for application in chemical and biochemical processes. ${ }^{8,9)}$

As part of the endeavor towards effective utilization of unused resources, the recovery of useful substances from food and agricultural wastes, such as wood meal, defatted rice bran, fish bones, etc., by environmentally friendly treatment has increasingly been attracting research interest. For instance, subcritical and supercritical water treatments have been investigated as a means to saccharify cellulose and hemicellulose for further fermentation to produce bioethanol. ${ }^{10-14)}$

Polysaccharides are one of the major constituents of biomass; they consist of non-electrolytic, cationic, and/or anionic saccharides. Hexuronic acids such as D-glucuronic, D-galacturonic, D-mannuronic, and Lguluronic acids are common building blocks of polyanionic saccharides, viz., pectin, heparin, hyaluronic acid, alginic acid, etc. Basic research on the degradation of hexuronic acids is of fundamental importance if one is to gain insight into the kinetics and mechanisms of degradation under subcritical conditions. Furthermore, this knowledge is also necessary for the recovery of these substances.

In recent years, though studies have been published on the kinetics of the degradation and hydrolysis of mono-, di-, oligo-, and polysaccharides in subcritical water, ${ }^{11,12,15-17)}$ application-oriented publications remain the majority. We too have reported several studies on the kinetics of degradation of mono- ${ }^{18)} \mathrm{di}_{-},{ }^{19-21)}$ tri- ${ }^{22)}$ and oligosaccharides $^{8)}$ under subcritical conditions. Nonetheless, the majority of our previously published studies covered only non-electrolytic saccharides. The goal of this study was to examine the degradation characteristics of glucuronic acid under subcritical conditions to determine in detail its degradation kinetics in subcritical water as the reaction medium. We carried out the degradation reaction of glucuronic acid under subcritical conditions at various temperatures in a continuous tubular reactor to characterize the reaction mechanism. Based on the results obtained, a possible degradation mechanism was proposed, and subsequently was validated.

\section{Materials and Methods}

Materials. D-Glucuronic acid or simply glucuronic acid (abbreviated GlcA, purity $>98 \%)$ and calcium chloride $\left(\mathrm{CaCl}_{2}\right)$ were purchased from Wako Pure Chemical Industries (Osaka, Japan). D-Glucurono-6,3lactone or simply glucuronolactone (GlcL, purity >99\%), Dowex 50WX8-400 ion-exchange resin, and 3-(trimethylsilyl)propionic2,2,3,3- $\mathrm{d}_{4}$ acid sodium salt $\left(\mathrm{TSP}-\mathrm{d}_{4}\right)$ were from Sigma-Aldrich Japan (Tokyo). Silicone oil was from Dow Corning Toray Silicone (Tokyo, Japan). Distilled water was used throughout the experiment.

\footnotetext{
$\dagger$ To whom correspondence should be addressed. Fax: +81-75-753-6285; E-mail: adachi@kais.kyoto-u.ac.jp
} 
Experimental setup. The degradation reaction was performed under subcritical conditions in a continuous tubular reactor, in which rapid heating of the feed solution and quenching of the degradation reaction could be achieved. The apparatus and experimental procedures were similar to those described in our previous studies. ${ }^{22,23)}$ The degradation experiments on GlcA were carried out in a continuous tubular reactor made of a stainless steel tube (SUS $316,0.8 \mathrm{~mm}$ i.d. $\times 1 \mathrm{~m}$ ). The coiled reactor was immersed in an R105HB silicone oil bath equipped with an MH-5E mini heater (Riko Kagaku Sangyo, Chiba, Japan). The reaction mixture leaving the reactor was passed through a cooling coil immersed in an ice bath. The reaction solutions were fed through the reactor with a Shimadzu LC-10AT $\mathrm{VP}_{\mathrm{V}}$ HPLC pump (Kyoto, Japan). The reaction system was maintained at a constant working pressure of $10 \mathrm{MPa}$ with a high pressure adjustable back-pressure valve (Upchurch Scientific, Oak Harbor, WA) installed at the end of the system.

Degradation reaction. Feed solutions of GlcA and GlcL were prepared by dissolving each of the solutes in distilled water at concentrations of $0.1,0.25,0.5$, and $1.0 \% \mathrm{w} / \mathrm{v}$. The solutions were degassed by ultrasonic vibration. The glass container from which the reaction solutions were fed was connected to a helium gasbag to prevent re-dissolution of oxygen into the solution throughout the degradation experiments. The solutions were delivered at a prescribed flow rate to give a specific residence time of 10 to $300 \mathrm{~s}$ in the reactor. The residence time was calculated from the flow rate, the inner volume of the reactor, and the water densities at room and degradation temperatures. The degradation experiments were performed at temperatures between 160 and $200^{\circ} \mathrm{C}$ at intervals of $10^{\circ} \mathrm{C}$. Approximately $2.5 \mathrm{ml}$ of the reactor effluent was collected into a sampling vessel for further analysis after about 10 times the length of the prescribed residence time had elapsed.

Concentration analysis of glucuronic acid and glucuronolactone. The collected reactor effluents were analyzed with a Shimadzu LC10AS HPLC equipped with a Shimadzu RID-10A refractive index detector to determine the remaining concentration of GlcA or GlcL. The columns used for separation were two Supelcogel Ca columns $(7.8 \mathrm{~mm}$ i.d. $\times 300 \mathrm{~mm}$, Sigma-Aldrich Japan) in series with a Supelguard Ca guard column $(4.6 \mathrm{~mm}$ i.d. $\times 50 \mathrm{~mm}$, Sigma-Aldrich Japan). The eluent was distilled water at a rate of $0.5 \mathrm{ml} / \mathrm{min}$. HPLC analysis was performed at room temperature at least in triplicate for every sample, and the values were averaged. The $\mathrm{pH}$ of the reactor effluents was also measured, at room temperature, with a Horiba F-13 pH meter (Kyoto, Japan).

Purification of glucuronolactone. Purification of glucuronolactone, which was formed during the treatment of GlcA under subcritical conditions, was performed in a glass column $(16 \mathrm{~mm}$ i.d. $\times 330 \mathrm{~mm})$ packed with Dowex 50WX8-400 ion-exchange resin. The resin was converted to calcium-ion form with a $2.0-\mathrm{mol} / 1 \mathrm{CaCl}_{2}$ solution until the $\mathrm{pH}$ of the effluent stabilized. Eight $\mathrm{ml}$ of the reactor effluent of GlcA was concentrated to an approximate volume of $2 \mathrm{ml}$ by rotary evaporation. Subsequently, the concentrated effluent was loaded onto the top of the packed bed of the resin and eluted with water at a flow rate of $0.5 \mathrm{ml} / \mathrm{min}$. The effluent was collected for every $5 \mathrm{~min}$ as a different fraction and then analyzed individually with the abovementioned HPLC system. The fraction that contained the main degradation product was lyophilized and subjected to further qualitative analyses by mass spectrometry (MS) and ${ }^{1} \mathrm{H}$ nuclear magnetic resonance $\left({ }^{1} \mathrm{H}\right.$ NMR).

$M S$ and ${ }^{1} H$ NMR analyses. The mass spectra of the purified samples were recorded on a Mariner electrospray ionization time-of-flight mass spectrometer (ESI-TOF-MS) (Applied Biosystems, Foster City, CA) coupled with a Hewlett-Packard 1100 series HPLC system (HewlettPackard, Palo Alto, CA). The electrospray ionization interface was operated in either the positive or the negative ion mode. The other interface settings, spray tip potential and nozzle potential, were set to $3,500 \mathrm{~V}$ and $150 \mathrm{~V}$ respectively. The two ion modes had the same set values.

${ }^{1} \mathrm{H}$ NMR was performed on a JEOL ECP500 NMR spectrometer (Tokyo) operating at $500 \mathrm{MHz}$ using $\mathrm{D}_{2} \mathrm{O}$ and $\mathrm{TSP}-\mathrm{d}_{4}$ as the solvent and internal standard respectively.

\section{Results and Discussion}

\section{Identification of glucuronolactone produced in the degradation of glucuronic acid}

The reaction solution at a feed GlcA concentration of $2.0 \% \mathrm{w} / \mathrm{v}$ decomposed at $160{ }^{\circ} \mathrm{C}$ for a residence time of $180 \mathrm{~s}$, was subjected to qualitative analysis by ESI-TOFMS. Based on the mass spectrum, one of the main degradation products of GlcA was suggested to be GlcL, based on the molecular mass of the compound of 176 . The compound was purified by ion-exchange chromatography using a packed column of Dowex 50WX8-400 ion-exchange resin. The purified compound was identified as GlcL by ${ }^{1} \mathrm{H}$ NMR. ${ }^{1} \mathrm{H}$ NMR $\left(500 \mathrm{MHz}, \mathrm{D}_{2} \mathrm{O}\right)$ : $\delta 5.48(1 \mathrm{H}, \mathrm{s}), 5.06-5.02(2 \mathrm{H}, \mathrm{m}), 4.75(1 \mathrm{H}, \mathrm{d}, J=$ $6.2 \mathrm{~Hz}), 4.37(1 \mathrm{H}, \mathrm{s})$.

\section{Degradation of glucuronic acid}

To detail the degradation reaction of GlcA further, the hydrolysis of GlcL, which was produced during the treatment of GlcA in subcritical water, was investigated over a temperature range of 170 to $200^{\circ} \mathrm{C}$. A peak of the same retention time as GlcA was recorded in the HPLC chromatograms of the reactor effluents, suggesting that the conversion of GlcA to GlcL occurs in a reversible manner. Dowben ${ }^{24)}$ reported the hydrolysis of GlcL to GlcA in distilled water even at a temperature as low as $37^{\circ} \mathrm{C}$, apparently supporting our results. The hydrolysis reaction was found to be accelerated by the presence of Tris and phosphate buffers. Under our tested reaction conditions, we supposed that GlcA (1) is converted to GlcL (2), as depicted in Fig. 1, through intramolecular hemiacetalization by nucleophilic addition of the $\mathrm{C} 3$ hydroxyl group to the aldehyde group and intramolecular esterification of the $\mathrm{C} 4$ hydroxyl group by the carboxylic acid group. The resulting GlcL was assumed to be further decomposed to other products, because a material balance that takes into account only GlcA and GlcL could not be obtained. Based on a fragmentary understanding of the reaction, we hypothesized a degradation mechanism for GlcA, as described in Fig. 2. By assuming all the steps to be first-order reactions, the kinetics of the degradation of GlcA and GlcL can thus be described by the equations below, which were numerically solved by the Runge-Kutta-Gill method:25)

$$
\begin{aligned}
& d C_{\mathrm{GlcA}} / d \tau=-\left(k_{\mathrm{AL}}+k_{\mathrm{Ad}}\right) C_{\mathrm{GlcA}}+k_{\mathrm{LA}} C_{\mathrm{GlcL}} \\
& d C_{\mathrm{GlcL}} / d \tau=k_{\mathrm{AL}} C_{\mathrm{GlcA}}-\left(k_{\mathrm{LA}}+k_{\mathrm{Ld}}\right) C_{\mathrm{GlcL}}
\end{aligned}
$$

where $C$ is the concentration and $\tau$ is the residence time. The rate constants, $k$, are subscripted with 'AL' and 'LA' for the reverse hydrolysis of GlcA to GlcL and the hydrolysis of GlcL to GlcA respectively, while the subscripts 'Ad' and 'Ld' indicate the degradation of GlcA and of GlcL respectively to the degradation products. $k_{\mathrm{AL}}, k_{\mathrm{LA}}, k_{\mathrm{Ad}}$, and $k_{\mathrm{Ld}}$ were estimated by minimizing the residual sum of squares between the values estimated by eqs. (1) and (2), and the experimental ones using Microsoft Excel Solver ${ }^{\circledR}$ for the degradations of GlcA and GlcL at a specific temperature. The rate constants determined for the degradation reaction using GlcA as substrate should be identical to their respective counterparts computed for the degradation of GlcL. 


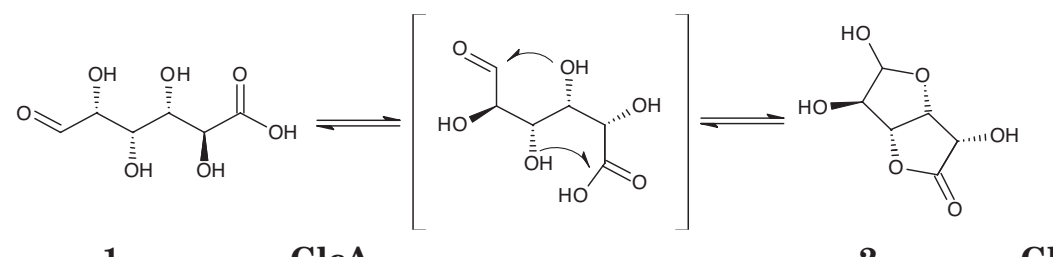

$1 \quad$ GlcA

2

GlcL

Fig. 1. Reaction Scheme for the Reversible Conversion of Glucuronic Acid (GlcA) to Glucuronolactone (GlcL).

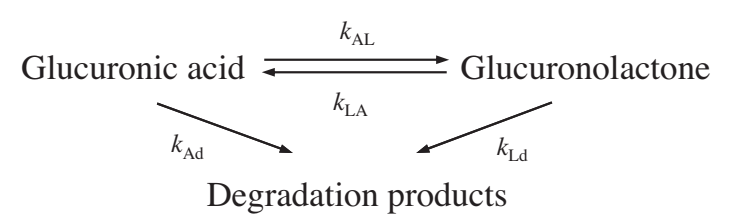

Fig. 2. Possible Reaction Scheme for the Degradation of Glucuronic Acid and That of Glucuronolactone in Subcritical Water.

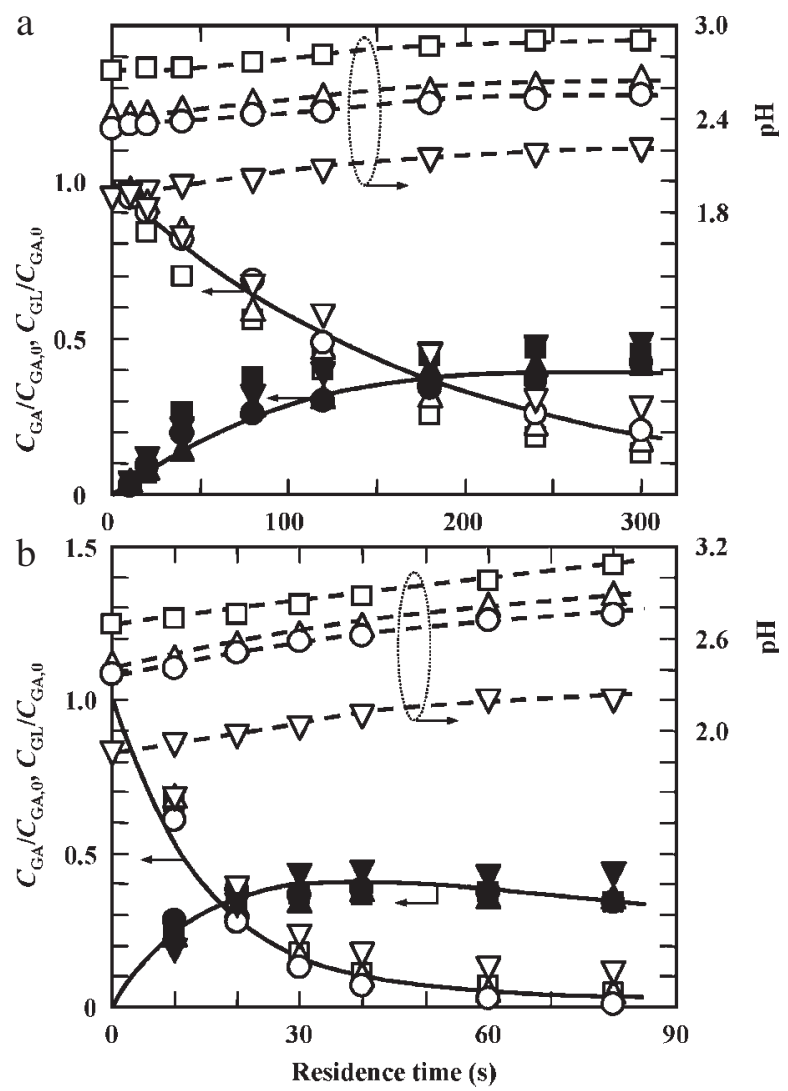

Fig. 3. Degradation of Glucuronic Acid at Different Feed Concentrations, $C_{\mathrm{GlcA}, 0}$, of $(\square, \boldsymbol{\square}) 0.1 \% \mathrm{w} / \mathrm{v},(\triangle, \boldsymbol{\Delta}) 0.25 \%$, $(\bigcirc, \bigcirc) 0.5 \%$, and $(\nabla, \boldsymbol{\nabla}) 1.0 \%$ at (a) $160^{\circ} \mathrm{C}$ and (b) $200^{\circ} \mathrm{C}$.

Open symbols indicate the concentration of glucuronic acid, $C_{\mathrm{GlcA}}$ and the $\mathrm{pH}$ of the reactor effluent, and the solid ones represent the concentration of glucuronolactone, $C_{\mathrm{GlcL}}$.

\section{Degradation of glucuronic acid at various feed} concentrations

Figures $3 \mathrm{a}$ and $\mathrm{b}$ show the relative concentrations of GlcA and GlcL of the reactor effluents to their respective feed GlcA concentrations, $C_{\mathrm{GlcA}, 0}$, over various residence times in the continuous tubular reactor and also their $\mathrm{pH}$ values at $160^{\circ} \mathrm{C}$ and $200^{\circ} \mathrm{C}$, respectively. Despite the difference in $C_{\mathrm{GlcA}, 0}$, all the plots for the concentration changes in GlcA and GlcL fell on a single curve at each temperature. The $C_{\mathrm{GlcA}, 0}$ dependency of GlcA degradation was negligible, and no significant

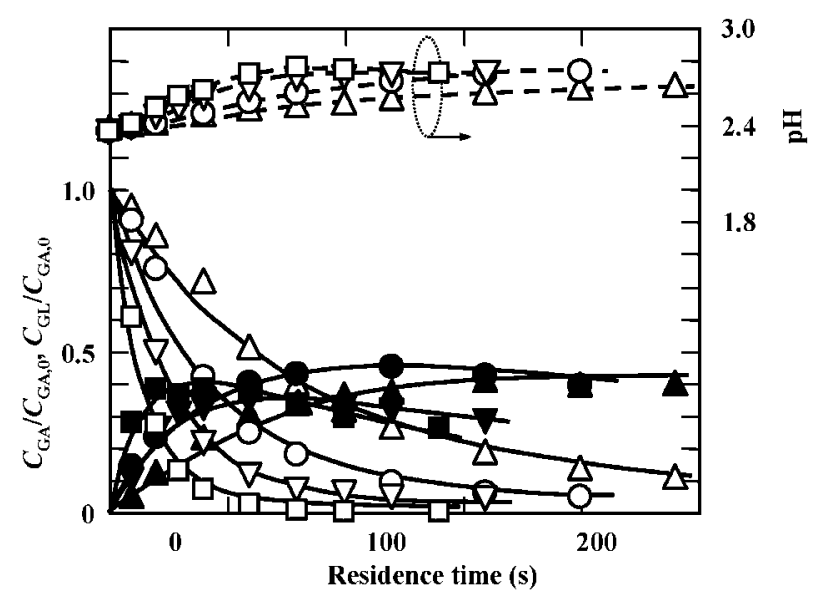

Fig. 4. Degradation Processes of $0.5 \% \mathrm{w} / \mathrm{v}$ Glucuronic Acid at $(\triangle, \boldsymbol{\Delta}) 170^{\circ} \mathrm{C},(\bigcirc, \boldsymbol{\odot}) 180^{\circ} \mathrm{C},(\nabla, \nabla) 190^{\circ} \mathrm{C}$, and $(\square, \boldsymbol{\square}) 200^{\circ} \mathrm{C}$. Open and solid symbols are defined as in Fig. 3.

difference was found among treatments that varied in $C_{\mathrm{GlcA}, 0}$. On the basis of this finding, we proceeded with further experiments at $C_{\mathrm{GlcA}, 0}$ of $0.5 \% \mathrm{w} / \mathrm{v}$. All the $\mathrm{pH}$ values of the reactor effluents were found to be higher for longer residence times, following a similar trend regardless of $C_{\mathrm{GlcA}, 0}$. However, the maximum changes recorded were merely magnitudes of less than 0.2 .

Degradation of glucuronic acid at various temperatures

Figure 4 presents the relative concentrations of GlcA and GlcL of the reactor effluents to $C_{\mathrm{GlcA}, 0}$ of $0.5 \% \mathrm{w} / \mathrm{v}$ over various residence times at degradation temperatures between 170 and $200^{\circ} \mathrm{C}$, and also the resulting $\mathrm{pH}$ changes. Solid curves were calculated using rate constants estimated to fit the experimental results at each temperature. Correlation with the models was very satisfactory for both GlcA and GlcL within the experimental temperature range, substantiating the validity of our proposed degradation mechanism (Fig. 2).

GlcA decomposed at a higher rate when the reaction temperature was higher. Dowben ${ }^{24)}$ mentioned that in the hydrolysis of GlcL, the GlcA/GlcL ratio at equilibrium was a temperature-dependent property. Presumably, the temperature dependency is also applicable to the reverse hydrolysis of GlcA, because the reaction is reversible. At higher temperatures, the GlcA concentration decreased at faster rates, which caused the $\mathrm{pH}$ values to increase, while the GlcL concentration increased in the corresponding order. Changes in the $\mathrm{pH}$ value were trivial. More noteworthy is the uneven material balances between GlcA and GlcL, which implies the presence of other degradation products.

Figure 5 shows the $\mathrm{pH}$ changes in the GlcA and the GlcL reaction solution as a function of their changes in 


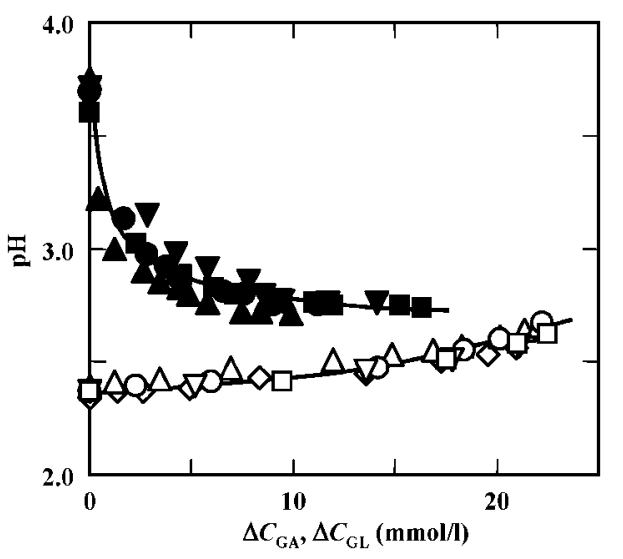

Fig. 5. Relationships between Changes in $\mathrm{pH}$ of the Reactor Effluents and the Amount of Consumed (open symbols) Glucuronic Acid, $\Delta C_{\mathrm{GlcA}}$, and (solid symbols) Glucuronolactone, $\Delta C_{\mathrm{GlcL}}$, at $170^{\circ} \mathrm{C}$, $180^{\circ} \mathrm{C}, 190^{\circ} \mathrm{C}$, and $200^{\circ} \mathrm{C}$.

The symbols are the same as in Fig. 4.

concentration. The changes in $\mathrm{pH}$ showed negligible dependency upon the degradation temperature. The $\mathrm{pH}$ value of the reaction solutions increased when more GlcA was lost to degradation, while a $\mathrm{pH}$ decrease was observed with increasing consumption of GlcL. The slight decrease in the GlcL concentration at the initial stage caused a drastic drop in $\mathrm{pH}$. Despite the small amounts of GlcA produced during the degradation of GlcL, GlcA demonstrated a strong effect upon $\mathrm{pH}$. The degradation of GlcA resulted in a comparably less significant rise in $\mathrm{pH}$. Even at the highest recorded consumption, the $\mathrm{pH}$ value showed only a modest increase, of approximately 0.3 . With a $\mathrm{p} K_{\mathrm{a}}$ value of about $2.9,{ }^{26)} \mathrm{GlcA}$ is a weak acid with a small extent of dissociation. Therefore, the observed $\mathrm{pH}$ changes are likely to be attributed to the comprehensive effects of other factors, including the other degradation products, although no further investigation was done.

\section{Degradation of glucuronolactone at various temper-} atures

The degradation of GlcL, which was produced during treatment of GlcA, was examined to study in detail the degradation of GlcA. The relative concentrations of GlcL and GlcA of the reactor effluents to the feed GlcL concentration, $C_{\mathrm{GlcL}, 0}$, of $0.5 \% \mathrm{w} / \mathrm{v}$ over various residence times at various degradation temperatures are shown in Fig. 6. The resulting $\mathrm{pH}$ changes are also included on the right $y$-axis. The solid correlation curves were those computed by fitting eqs. (1) and (2) to the experimental data. Satisfactory correlation of the data with the models again validated the proposed degradation mechanism.

Resembling the trend observed in the degradation of GlcA, GlcL also decomposed at a higher rate at higher degradation temperatures. However, GlcA was produced as a main degradation product at rates almost indistinguishable among the treatments at different degradation temperatures. The $\mathrm{pH}$ values dropped remarkably, particularly at $200{ }^{\circ} \mathrm{C}$, in treatments with residence times shorter than $100 \mathrm{~s}$, but stayed almost unchanged thereafter. Uneven material balances between GlcA and GlcL were also clearly seen.

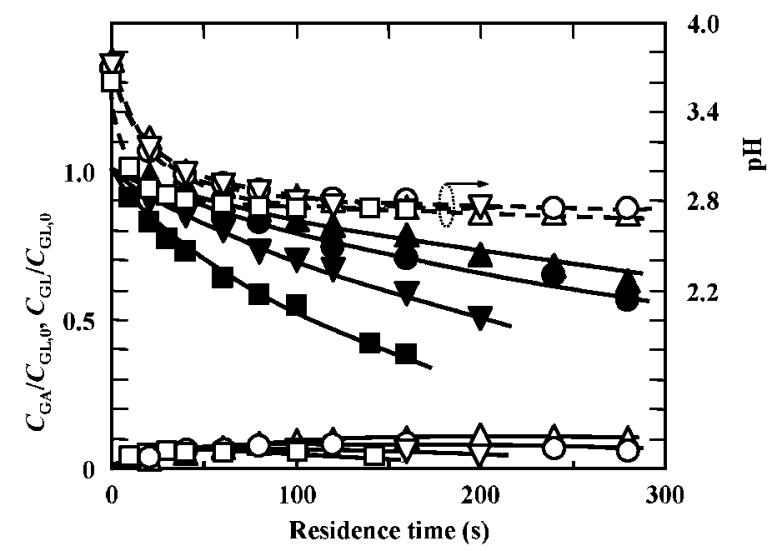

Fig. 6. Degradation Processes of $0.5 \% \mathrm{w} / \mathrm{v}$ Glucuronolactone at $170^{\circ} \mathrm{C}, 180^{\circ} \mathrm{C}, 190^{\circ} \mathrm{C}$, and $200^{\circ} \mathrm{C}$.

Open and solid symbols are defined as in Fig. 4. The subscript 0 represents the feed.

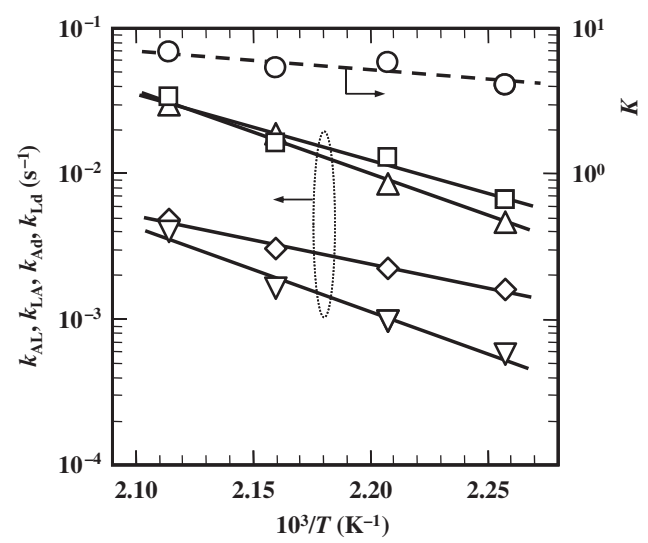

Fig. 7. Arrhenius Plots for the Rate Constants, $(\square) k_{\mathrm{AL}},(\diamond) k_{\mathrm{LA}},(\triangle)$ $k_{\mathrm{Ad}}$, and $(\nabla) k_{\mathrm{Ld}}$, for the Degradation of Glucuronic Acid and Glucuronolactone, and the Temperature Dependence of the Reaction Equilibrium Constant, $K$, between Glucuronic Acid and Glucuronolactone.

The definitions of the rate constants are given in Fig. 2, and $K$ is defined by eq. (4).

\section{Temperature dependence of the rate constants}

Degradation of GlcA and GlcL was observed at different temperatures for various residence times. Figure 7 shows the plots of the rate constants and equilibrium constant, $K$, versus the reciprocal of the absolute temperature, $1 / T$. Because the rate constants, $k$, obey the Arrhenius relationship, the temperature dependence of $k$ could be expressed by the Arrhenius relation, as

$$
k=k_{0} \exp (-E / R T)
$$

where $E$ is the activation energy, $k_{0}$ is the frequency factor, and $R$ is the gas constant. The $E$ and $k_{0}$ values were determined from the Arrhenius plot and are tabulated in Table 1. The $E$ values for the reverse hydrolysis of GlcA to GlcL and that of the hydrolysis of GlcL to GlcA were 88.5 and $63.2 \mathrm{~kJ} / \mathrm{mol}$ respectively. GlcA and GlcL were more thermolabile than glucose, which was degraded to a lesser extent, at a temperature of $200{ }^{\circ} \mathrm{C}$ for instance. ${ }^{18)}$ Our previous study of the degradation of D-galacturonic acid revealed a similar result; uronic acid was more susceptible to degradation than the corresponding hexose in subcritical water. ${ }^{23)}$ 
Table 1. Activation Energies $E$ and Frequency Factors $k_{0}$ for the Reaction Steps Involved in the Degradation of Glucuronic Acid and of Glucuronolactone Determined by the Arrhenius Plots

\begin{tabular}{lcccc}
\hline \hline $\begin{array}{c}\text { Reaction } \\
\text { step }^{\mathrm{a}}\end{array}$ & $\mathrm{AL}$ & LA & $\mathrm{Ad}$ & $\mathrm{Ld}$ \\
\hline $\begin{array}{l}E[\mathrm{~kJ} / \mathrm{mol}] \\
k_{0}[1 / \mathrm{s}]\end{array}$ & $\begin{array}{c}88.5 \\
1.86 \times 10^{8}\end{array}$ & $4.43 \times 10^{4}$ & $5.24 \times 10^{10}$ & $4.90 \times 10^{9}$ \\
\hline
\end{tabular}

${ }^{\mathrm{a}}$ The notations are defined as in Fig. 2.

Further, the $E$ value for the degradation of GlcA to other degradation products was computed to be $110 \mathrm{~kJ} / \mathrm{mol}$, similar to that determined for GlcL. These values are in the same order of magnitude as the $E$ values for the degradation of D-galacturonic acid and sodium galacturonate. ${ }^{23)}$ The equilibrium constant, $K$, was defined by the following equation:

$$
K=k_{\mathrm{AL}} / k_{\mathrm{LA}}
$$

The temperature dependence of $K$ indicates the obedience to van't Hoff's law (Fig. 7), and thus could be expressed by van't Hoff's equation:

$$
d \ln K / d(1 / T)=-\Delta H / R
$$

The reversible conversion of GlcA to GlcL was an endothermic reaction, with the enthalpy change, $\Delta H$, computed to be $25.4 \mathrm{~kJ} / \mathrm{mol}$. The temperature dependence of the equilibrium constant indicated a shift in the equilibrium of reverse hydrolysis of GlcA to GlcL under subcritical conditions at higher temperatures.

\section{Conclusions}

The degradation of GlcA under subcritical conditions in a continuous tubular reactor was studied kinetically, and the hypothesized degradation mechanism was confirmed. The degradation reaction was found to be temperature-dependent, and the rate constant of each proposed reaction step was satisfactorily expressed by the Arrhenius equation. The degradation of GlcA was an endothermic process that proceeded at a higher rate with increasing heat supply. The understanding of the degradation mechanism of GlcA established in this study is expected to be useful for the efficient recovery of hexuronic acids, in particular GlcA, from agricultural or food wastes by subcritical water treatment. In view of the fact that protein usually accounts for at least part of agricultural and food wastes, an investigation of the degradation of dipeptides is in progress.

\section{Acknowledgments}

This study was financially supported by the Cooperation for Innovative Technology and Advanced Re- search in Evolutional Area (CITY AREA) program of the Ministry of Education, Culture, Sports, Science, and Technology of Japan. We are grateful to Dr. Y. Kimura of Okayama University for his contribution to the project during his tenure with our division. One of the authors, R.W., thanks the Japanese government for a Monbukagakusho Scholarship.

\section{References}

1) Clifford T, "Fundamentals of Supercritical Fluids," Oxford University Press, New York, pp. 1-23 (1999).

2) Ayala RS and Castro L, Food Chem., 75, 109-113 (2001).

3) Herrero M, Cifuentes A, and Ibañez E, Food Chem., 98, 136148 (2006).

4) Wiboonsirikul J, Kimura Y, Kadota M, Morita H, Tsuno T, and Adachi S, J Agric. Food Chem., 55, 8759-8765 (2007).

5) Krammer P and Vogel H, J. Supercrit. Fluids, 16, 189-206 (2000).

6) Kuhlmann B, Arnett EM, and Siskin M, J. Org. Chem., 59, 3098-3101 (1994).

7) Lesutis HP, Gläser R, Liotta CL, and Eckert CA, Chem. Commun., 20, 2063-2064 (1999).

8) Haghighat Khajavi S, Ota S, Kimura Y, and Adachi S, J. Agric. Food Chem., 54, 3663-3667 (2006).

9) Wiboonsirikul J and Adachi S, Food Sci. Technol. Res., 14, 319-328 (2008).

10) Sasaki T, Shibata M, Miki T, Hirosue H, and Hayashi N, Energy Fuels, 10, 684-688 (1996).

11) Sasaki M, Fang Z, Fukushima $Y$, Adschiri $T$, and Arai K, Ind. Eng. Chem. Res., 39, 2883-2890 (2000).

12) Sasaki T, Shibata M, Sumi T, and Yasuda S, Ind. Eng. Chem. Res., 41, 661-665 (2000).

13) Ehara K, Saka S, and Kawamoto H, J. Wood Sci., 48, 320-325 (2002).

14) Ehara K and Saka S, J. Wood Sci., 51, 148-153 (2005).

15) Kabyemela BM, Adschiri T, Malaluan RM, and Arai K, Ind. Eng. Chem. Res., 36, 1552-1558 (1997).

16) Kabyemela BM, Takigawa M, Adschiri T, Malaluan RM, and Arai K, Ind. Eng. Chem. Res., 37, 357-361 (1998).

17) Sasaki M, Furukawa M, Minami K, Adschiri T, and Arai K, Ind. Eng. Chem. Res., 41, 6642-6649 (2002).

18) Haghighat Khajavi S, Kimura Y, Oomori T, Matsuno R, and Adachi S, J. Food Eng., 68, 309-313 (2005).

19) Haghighat Khajavi S, Kimura $Y$, Oomori $T$, Matsuno R, and Adachi S, Biosci. Biotechnol. Biochem., 68, 91-95 (2004).

20) Haghighat Khajavi S, Kimura Y, Oomori T, Matsuno R, and Adachi S, LWT-Food Sci. Technol., 38, 297-302 (2005).

21) Oomori $T$, Haghighat Khajavi S, Kimura $Y$, Adachi $S$, and Matsuno R, Biochem. Eng. J., 18, 143-147 (2004).

22) Haghighat Khajavi S, Ota S, Nakazawa R, Kimura $Y$, and Adachi S, Biotechnol. Prog., 22, 1321-1326 (2006).

23) Wang R, Kobayashi T, and Adachi S, J. Appl. Glycosci., 56, 181-184 (2009).

24) Dowben RM, Biochim. Biophys. Acta, 31, 454-458 (1959).

25) Press WH, Teukolsky SA, Vetterling WT, and Flannery BP, "Numerical Recipes in $\mathrm{C}++$," Cambridge University Press, Cambridge, pp. 715-719 (2002).

26) Wang HM, Loganathan D, and Linhardt RJ, Biochem. J., 278, 689-695 (1991). 